Voix et Images

\title{
L'oreille enchantée. Le corps imaginaire de la parole chez Claude Gauvreau
}

\section{Jacques Cardinal}

Volume 21, numéro 3 (63), printemps 1996

Le bavardage dans la littérature québécoise

URI : https://id.erudit.org/iderudit/201262ar

DOI : https://doi.org/10.7202/201262ar

Aller au sommaire du numéro

Éditeur(s)

Université du Québec à Montréal

ISSN

0318-9201 (imprimé)

1705-933X (numérique)

Découvrir la revue

Citer cet article

Cardinal, J. (1996). L’oreille enchantée. Le corps imaginaire de la parole chez Claude Gauvreau. Voix et Images, 21(3), 520-543.

https://doi.org/10.7202/201262ar
Résumé de l'article

Résumé

L'auteur analyse ici la poétique du texte gauvrien à partir d'une théorie psychanalytique du langage et du nom. Il cherche à montrer le lien entre une textualité de la destruction et de la réinvention de la langue et un imaginaire du corps chimérique. 


\title{
L'oreille enchantée. Le corps imaginaire de la parole chez Claude Gauvreau *
}

\author{
Jacques Cardinal, Université de Montréal
}

\begin{abstract}
L'auteur analyse ici la poétique du texte gauvrien à partir d'une théorie psychanalytique du langage et du nom. Il cherche à montrer le lien entre une textualité de la destruction et de la réinvention de la langue et un imaginaire du corps chimérique.
\end{abstract}

Jadis, les cigales étaient des hommes, ceux qui existèrent avant que naissent les Muses. Puis, quand les Muses furent nées et que leur chant eut commencé de se faire entendre, certains des hommes de ce temps-là furent, raconte-t-on, à ce point mis par le plaisir hors d'eux-mêmes que de chanter leur fit négliger de manger et de boire, si bien qu'ils moururent sans s'en apercevoir. C'est de ces hommes que, par la suite, a surgi la race des cigales ${ }^{1} \ldots$

Bien entendu, c'est Socrate qui parle, et l'on sait à quel point il se méfie de la force séductrice du chant et veut mettre de l'ordre dans l'usage du discours. Cependant, il y a là une intuition quant au statut de la parole qui ne cesse de se poser dans nombre de récits et qui concerne cette force du chant pouvant être tout autant bénéfique que maléfique pour celui ou celle qui l'entend. Ainsi, dans L'Odyssée, le valeureux Ulysse a-t-il choisi d'être attaché au mât de son navire pour ne pas être fatalement emporté par le chant ensorceleur des sirènes qu'il veut tout de

* Cette étude s'inscrit dans le cadre d'une recherche subventionnée par le Conseil de recherche en sciences humaines du Canada.

1. Platon, Phèdre, traduction, introduction et notes de Luc Brisson, Paris, UGE, coll. "Garnier-Flammarion ", 1989. 
même entendre, lequel promet "un lourd trésor de science ${ }^{2}$ ". Mais ce chant, nous lecteur, nous ne l'entendrons jamais, puisque déjà le bateau d'Ulysse s'éloigne de l'île et que les sirènes ne chantent que la promesse d'un chant absolument inouï. Ulysse, en définitive, n'entend que l'appel de ce chant inouï qui le restera à jamais: Ulysse a été prévenu par Circé, la royale, du danger qui le menaçait.

Ce récit des sirènes serait ainsi comme une rêverie sur le langage promettant un absolu ravissement, un savoir absolu par où le sujet maittriserait enfin sa destinée; c'est là, on s'en souviendra, le contraire de la condition d'Ulysse qui a besoin de toute sa ruse, doué qu'il est de mètis, pour triompher de l'adversité et de l'empiricité quasi imprévisible du monde ${ }^{3}$. Le chant des sirènes se situe donc à l'extrémité d'un monde dominé par des forces contradictoires, dominé par le polémos, et l'on peut dire qu'il est l'envers de ce monde puisqu'il promet d'une certaine manière le règne de l'unisson contre celui de la discorde où le malheur des hommes est parfois peu de chose sous le regard des dieux. Or, il est remarquable que ce chant, qui se veut promesse de savoir absolu en même temps que caresse irrésistible, masque en définitive le visage de la mort. Comme si, dans la rêverie de cette coïncidence entre savoir et langage conduisant le sujet à un sentiment de plénitude, la mort se dévoilait ultimement comme étant l'obscur maitre de ce désir. L'inouï de la plénitude que recèle le chant serait donc, dans ce récit, l'égal d'une rencontre avec la mort, soit la fin, pour le sujet, d'un monde déterminé par l'expérience du manque et de la différence ${ }^{4}$.

Ce récit affirme explicitement, certes, que le chant est un pouvoir, c'est-à-dire une certaine forme de langage qui peut s'emparer du corps, le maîtriser, le posséder, et lui faire violence. Or, s'il le peut, c'est que, d'entrée de jeu, le chant est un corps qui, en se faisant caresse, touche l'oreille. Le chant nous touche, nous émeut, vibre en nous. Cette voix chantante caressant l'oreille, c'est déjà la rencontre de deux corps jouant du jeu intermittent du son et du silence, intermittence qui est là comme le premier battement d'un désir ou d'un rapport à l'autre. En cela, on pourrait dire que le chant est ce corps de désir offert à l'autre dans la voix d'une sirène ou d'un poète. L'oreille et le chant font ainsi corps pour un sujet déterminé par le désir ${ }^{5}$. La médecine de ce temps traitait d'ailleurs par la musique et la prosodie, sinon par le théâtre de type cathartique, les

2. Homère, L'Odyssée, traduction, notes et postface de Philippe Jaccottet, Paris, François Maspero, coll. "La Découverte", 1982, p. 203.

3. Marcel Detienne, Jean-Pierre Vernant, Les Ruses de l'intelligence. La mètis des Grecs, Paris, Flammarion, coll. "Champs", 1974.

4. À relire en ce sens les très belles pages que Maurice Blanchot consacre au chant des sirènes dans Le Livre à venir, Paris, Gallimard, coll. "Idées ", 1959, p. 9-40.

5. Sur cette question, je renvoie notamment le lecteur au livre de Denis Vasse, L'Ombilic et la Voix. Deux enfants en psychanalyse, Paris, Seuil, coll. "Le champ freudien", 1974, 
sujets a-rythmiques; cette médecine reconnaissait ainsi une homologie entre le battement du cour et l'eurythmie de la prosodie ${ }^{6}$.

Ce récit nous apprend également que, si le corps peut être possédé par un chant, cette violence peut cependant prendre une forme qui en est le contraire dans la mesure où le chant, de prime abord, ne serait qu'une simple caresse de l'oreille masquant le travail d'une autre violence. Que la caresse du chant puisse être violence, voilà un paradoxe qui nous révèle que le langage est, à l'origine, travaillé par une indétermination radicale et que le sujet est, pour ainsi dire, condamné à l'interprétation parfois contradictoire des signes. Il y aurait donc là comme une duplicité originaire du langage, un jeu, un écart, et une différence par où le sujet ferait l'épreuve de l'interprétation. Et c'est cette indétermination justement que la philosophie de Platon cherchera à arraisonner en décrivant, autant que faire se peut, l'ordre du discours. Ce que donne à lire ce récit, c'est donc ce savoir déjà ancien que la parole est une arme à double tranchant; elle permet certes au sujet de nommer le_monde et de le maitriser comme c'est d'ailleurs le cas pour le poète des poètes, Orphée, mais elle peut aussi maîtriser le sujet, l'ensorceler, l'envoûter, le posséder. En cela, et par le langage, le sujet fait l'expérience d'un vertige dans la mesure où l'origine qui le fonde dans la parole ne peut être assignable.

Sans doute est-il significatif, enfin, que ce chant soit chanté par une chimère, une créature mi-femme mi-poisson, confirmant par là que ce chant du savoir absolu est au-delà ou en deçà de l'humaine condition, laquelle est assujettie à l'incertitude du devenir. Tout se passe, en effet, comme si le corps chantant du savoir absolu ne pouvait être qu'une chimère là où, non sans paradoxe, des corps hétérogènes se nouent ensemble à l'instant même où le chant promet le récit d'un monde transparent et homogène. C'est dire aussi que ce chant inouï se soutient déjà d'un corps imaginaire où le désir du savoir absolu se confond avec l'énigme angoissante du désir et de la différence sexuelle. Cette sirène serait donc l'imaginaire de cette différence, soit l'incarnation de l'hétérogénéité insurmontable à l'œuvre dans le monde. Que la toute-puissance de ce chant inouï s'incarne dans une voix de femme nous rappelle aussi la persistance d'un fantasme où la féminité est investie d'un supposé savoir sur le monde et le désir. La toute-puissance de cette voix de femme s'avère en fait, pour Ulysse, tout autant menaçante que protectrice puisque la déesse Athéna, dont l'œil étincelle, guide ses pas vers Ithaque. Certes, L'Odyssée est un récit où prédomine (encore) le monde de

p. 183-218. Voir aussi son article, "La voix qui crie dans le désêtre", Esprit, juillet 1980, p. $63-80$.

6. Jackie Pigeaud, Folie et cures de la folie chez les médecins de l'Antiquité gréco-romaine (La manie), Paris, Société d'édition "Les Belles lettres", "Collection d'études anciennes", 1987, p. 155-162. 
l'anthropomorphisme et de l'animisme - tout ce que Platon, d'ailleurs, condamnera notamment dans la République en arguant de ce que doit être le bon usage du discours et de la mimèsis —, mais la littérature, justement, n'a peut-être jamais cessé de jouer de la prosopopée afin d'affirmer une autre lecture du monde fondée sur l'incertitude des frontières entre les mots et les choses ${ }^{7}$.

Dans le mythe des cigales évoqué par Socrate, on constate aussi que le chant peut devenir absolu ravissement, qu'il peut enchanter l'oreille jusqu'à ce que le sujet en vienne à oublier les conditions de sa finitude et de son existence. Cet absolu ravissement serait aussi, en même temps que l'expérience de la plénitude, la mort du sujet; comme si, encore une fois, la plénitude du chant coïncidait avec la fin de l'épreuve du monde en tant que cette épreuve est celle du manque, de la séparation, de la souffrance et de la jouissance, de l'altérité et du temps. Cette oreille enchantée, ravie, béate serait celle d'un sujet qui ne se possède plus, mourant paradoxalement dans l'euphorie et non dans les souffrances de l'agonie. Pour ne pas céder à cette caresse maléfique du chant, Socrate en appelle ainsi à la vigilance, à la lucidité, à l'ironie du discours philosophique pour maitriser ce corps de langage.

Or, depuis Platon du moins, on a opposé la basse matérialité du corps avec la sagesse idéale d'un esprit dont la voix se devait d'être le relais diaphane ${ }^{8}$. Cette voix sublime et sublimée se voulait égale à l'idéalité de la vérité philosophique contemplée s'arrachant enfin à l'empiricité du monde. Cette voix - la matérialité de cette voix - était ainsi transfigurée ou arraisonnée à une méta-physique qui, pour se donner tout entière à la contemplation de l'Idée, voulait effacer les aspérités, le ton, le timbre, le grain de la voix qui donnent aux mots et à la phrase son rythme, son accent, son phrasé par où justement se révèle un corps, c'està-dire un sujet aux prises avec le monde et le langage à interpréter. En bref, le sujet de l'énoncé de la vérité aurait voulu effacer le sujet de l'énonciation, ce sujet de la finitude parlant, depuis son corps, nœud de désir et de mort qui le livre au monde et à la rencontre de l'autre. Ce discours sur le langage a pu inciter Platon, on s'en souviendra aussi, à

7. Voir Platon, La République, livre 2, 3, et 10, texte établi et traduit par Émile Chambry, Paris, Les Belles Lettres, "Collection des Universités de France", 1932. Voir aussi, sur cette question de la mimèsis, Antonia Soulez, La Grammaire philosophique chez Platon, Paris, Presses universitaires de France, coll. "Philosophie d'aujourd'hui", 1991, p. 72110.

8. J'évoque ici l'analyse du phonocentrisme et du logocentrisme faite par Jacques Derrida, De la grammatologie, Paris, Minuit, coll. "Critique", 1967; de même "La pharmacie de Platon", La Dissémination, Paris, Seuil, coll. "Tel Quel ", 1972. Bien que ce phonologocentrisme soit l'idéal du discours philosophique, il n'en demeure pas moins que Platon a conscience de la médiation obligée du sensible, de l'écriture, dans l'élaboration de la pensée. Le livre de Pierre Gaudin, Platon et l'alphabet (Paris, Presses universitaires de France, coll. "Philosophie d'aujourd'hui ", 1990), le montre bien. 
exclure le poète de la République fondée sur la suprématie d'un jeu de langage, la dialectique, contre les égarements sophistiques et les effets pervers de la re-présentation où l'art n'est plus que la copie d'une copie 9 . On peut certes déconstruire ce discours platonicien, comme on le dit aujourd'hui, pour y reconnaître un sujet qui, voulant gommer la phénoménalité du corps, le sublime malgré tout dans le corps parfait des figures de la géométrie, dans une architecture soumise au nombre d'or, ou se laisse bercer par la musique des sphères ${ }^{10}$. Harmonie préétablie que le philosophecontemplateur retrouve, ou plus exactement se remémore, depuis le mathématique, le ta mathemata que Heidegger analyse justement comme le travail de dévoilement de ce que le sujet sait déjà depuis toujours ${ }^{11}$. Tout se passerait donc dans ce cas comme si la voix, le sujet de l'énonciation, disparaissait pour que se laisse entendre la voix transparente de la vérité; ventriloque d'une voix immémoriale et tutélaire où le sujet surmonterait sa finitude dans le monde. Cette question de l'existence d'une voix de la vérité a ainsi été reliée à une certaine vision de la nature, nature qui justement n'était pas appréhendée dans son opposition à la culture.

Or, notre sensibilité à nous, maintenant, est très souvent à l'opposé de cela, tant il est vrai que nous vibrons à la voix, au style, à l'interprétation, bref, à la singularité qui s'élève pour chanter ce qu'il y a encore d'inouï dans le monde. En cela, l'esthétique, l'art, a pu s'élaborer, par-delà tous les projets de réconciliation entre l'éthique et la vérité métaphysicienne, comme le lieu d'une continuelle expérimentation du sujet à l'égard du sens. Mais, entre le vertige tout autant euphorique que dysphorique d'un sujet qui expérimente tous les possibles de la signification en jouant sur la matérialité même de la langue, persiste, comme toujours, la question de la limite et de la communicabilité de l'œuvre d'art. Ainsi, il y a des ouvres plus inquiètes et peut-être plus inquiétantes encore en ce qu'elles soumettent le langage à un travail d'écriture où celui-ci, de transitif qu'il était, s'opacifie ou creuse sa propre matière pour laisser résonner l'écho d'une autre profondeur. L'œuvre de Gauvreau, on le verra, tout comme celle d'Antonin Artaud, par exemple, est de celles-là ${ }^{12}$. Si, en peinture, Cézanne et Manet ont pu diversement laisser apparaître peu à peu, et par-delà le signifié ou l'anecdote, la matière plastique ou la pâte des couleurs, on peut dire aussi qu'un Mallarmé, cédant l'initiative aux

9. Platon, La République, livre 10, op. cit.

10. Voir Platon, Timée, traduction, introduction et notes de Luc Brisson, Paris, UGE, coll. "Garnier-Flammarion", 1992. Voir aussi Matila C. Ghyka, Le Nombre d'or, Paris, Gallimard, 1959.

11. Martin Heidegger, Qu'est-ce qu'une chose?, traduit par Jean Reboul et Jacques Taminiaux, Paris, Gallimard, coll. "Classiques de la philosophie", 1971, p. 81-88.

12. L'œuvre de Gauvreau est proche aussi du futurisme d'un Marinetti, du lettrisme d'un Isidore Izou, ou encore d'un Khlebnikov. Voir Jean-Pierre Denis, "Glossolalie, langue universelle, poésie sonore ", Langages (Les glossolalies), nº 91, septembre 1988, p. 75-104. 
mots qui s'allument de reflets réciproques, a donné au signifiant une profondeur jusque-là inouïe ${ }^{13}$. Or, cette importance donnée au signifiant dans la littérature fait partie d'une très ancienne rêverie cratylienne sur la motivation du nom, rêverie dont Gérard Genette a montré le fantasme insistant tout au long de notre histoire littéraire ${ }^{14}$. L'œuvre de Gauvreau se situe dans la mouvance de cette histoire touchant la langue et le discours de la représentation, et plus particulièrement la littérature en tant que lieu où le signifiant se manifeste dans son épaisseur, la rêverie sur le nom continuant de s'y énoncer comme un discours de motivation.

Le mythe des cigales, évoquant ce qu'il peut y avoir de fascination mortelle dans l'écoute du chant, révèle aussi, depuis notre savoir de la psychanalyse, un sujet capté par la jouissance de son propre babil. La déclamation gauvrienne laisse peut-être entendre quelque chose de cette captation mortifère du sujet dans le chant en se faisant exploration à la fois triomphante et véhémente du langage comme lieu paradigmatique du procès identitaire et différentiel qui fonde le sujet. L'œuvre de Gauvreau ne cesse à sa manière d'ouvrir la question de ce qui peut être entendu d'un chant (l'exploréen) qui se fait à la fois destruction et réinvention de la langue. On retrouve en effet chez Gauvreau un vertige semblable à ceux qui sont évoqués dans les récits des sirènes et des cigales, dans la mesure où il s'agit encore, malgré la parade triomphante de cette écriture, d'une captation mortifère dans la langue. L'écriture de Gauvreau, on le sait, est à la limite de la lisibilité (si l'on entend par là un certain protocole par où le sujet s'entend, grosso modo, à déchiffer le sens de ce qu'il lit), puisqu'elle pousse le lecteur à une interrogation radicale sur la valeur signifiante du langage. Ainsi, comment lire, interpréter, de quelle oreille nous convient-il d'entendre ou pas ce qui s'écrit dans ce poème qui clôt les Euvres créatrices complètes, dernier poème-de Jappement à la lune:
ghédérassann omniomnemm wâkulé orod écmon zdhal irchpt laugouzou- gldefterrpanuclémenpénucleptadussel ferf folfoufaulô farmurerr a clô dzorr ${ }^{15}$

Comment lire cela, et selon le ton qu'on y met, c'est-à-dire selon des effets de corps qui déjà interprètent ce magma de consonnes et de voyelles, sans ressentir d'emblée un sentiment d'étrangeté, d'absurdité et

13. “L'œuvre pure implique la disparition élocutoire du poëte, qui cède l'initiative aux mots, par le heurt de leur inégalité mobilisés; ils s'allument de reflets réciproques comme une virtuelle traînée de feux sur des pierreries, remplaçant la respiration perceptible de l'ancien souffle lyrique ou la direction personnelle enthousiaste de la phrase". Stéphane Mallarmé, CEuvres complètes, Paris, Gallimard, coll. "La Pléiade", 1945 , p. 366.

14. Gérard Genette, Mimologiques. Voyage en Cratylie, Paris, Seuil, coll. "Poétique", 1976.

15. Claude Gauvreau, Euvres créatrices complètes, Montréal, Parti Pris, coll. "Chien d'or", 1977, p. 1498. Les renvois à ce texte seront désormais identifiés par le sigle $O C$, suivi du folio. 
d'incongruité confinant le lecteur à l'angoisse ou au fou rire (ce qui est peut-être du reste et dans certains cas la même chose), sinon à l'indifférence? Comme si le lecteur, libéré un instant du procès référentiel de la langue, pouvait projeter sur ce magma les différents récits de ses associations fantasmatiques. De telle sorte qu'à un premier niveau, ce magma apparait bel et bien comme un corps, dans la mesure où nous sommes liés à lui par un procès d'identification d'où nous cherchons à l'interpréter. Que ce magma puisse nous parler selon qu'en le lisant on éprouve du ravissement, de l'angoisse, de la haine ou de l'indifférence, voilà qui situe notre corps à la rencontre d'un autre corps. C'est en cela, pourrait-on dire, que le corps est imaginaire puisqu'il se saisit, s'invente et se masque depuis ce qui le fait sujet de l'identification, c'est-à-dire sujet dont le procès identitaire est déterminé par le jeu de la spécularisation. Sinon, comment pourrions-nous expliquer que nous puissions lire, nous arrachant plus ou moins à nous-mêmes, tant de récits où nous nous identifions à tel ou tel héros ou anti-héros? Que nous puissions nous identifier, par-delà la contingence de notre inscription sexuelle et historique, à un homme, une femme, un animal ou un objet quelconque montre que le sujet vit aussi de cette labilité ou plasticité de l'imaginaire. C'est pourquoi on pourrait dire que le corps imaginaire est celui que le sujet se donne au fil de ses identifications avec l'autre, parce qu'il ne se saisit qu'à travers une image — des images - plus ou moins stable et stabilisante de son désir.

En prenant ici pour point de départ l'œuvre de Gauvreau, je voudrais réfléchir sur un certain imaginaire du corps qui, touchant dès le départ une représentation déterminée du langage, engendre des récits très souvent traversés par le corps chimérique, monstrueux ou mal symbolisé. Je voudrais donc tenter de lire, depuis une certaine poétique de la langue gauvrienne, la construction imaginaire du corps qui insiste dans cette œuvre, ce qui, dans la poétique de l'exploréen, est conséquent avec l'imaginaire du corps chimérique tel que nous pouvons le lire notamment dans La Ventouse anonyme. Aussi, ce qui, dans ce rapport à la langue, signale la double scène du poète incompris, maudit par la société, et l'annonce d'un autre langage consacrant le règne d'un nouvel égrégore (ou d'une autre forme de civilisation). Cette langue inouïe n'estelle pas aussi l'appel d'un nom capable de trancher dans le babil exploréen?

\section{L'exploréen ou le babil triomphant et véhément de la différence}

Julia Kristeva a tenté, relisant Freud depuis Husserl et surtout Lacan, un récit des origines du langage basé sur l'opposition entre la chora et le thétique (le Symbolique et l'ordre nominal) ${ }^{16}$. La chora désigne ce fond

16. Julia Kristeva, La Révolution du langage poétique, Paris, Seuil, 1974, p. 17-99. 
pulsionnel où les rythmes de ruptures et d'articulations sont les premières manifestations - ordonnancements - d'un corps qui ne s'est pas encore constitué comme sujet sur le plan du Symbolique ou de la Loi. Le frayage pulsionnel s'articule ainsi selon ces premiers effets de différences pour former des nœuds, des traces, des continuités et des discontinuités. Il est aussi, en tant que lieu de scissions permanentes entre charges et stases sans identité, le lieu d'un processus où prédomine la négativité (ou la pulsion de mort). Ce n'est pas encore l'ordre du nom, du signe et de la syntaxe mais celui, plus musical, du rythme comme effet de discernement ou de différenciation. En ce sens, chora désigne ce fond de tensions et de forces pré-œdipien sur lequel viendra cependant s'élaborer le procès de la signifiance qui fera de ce corps un sujet détaché de la mère archaïque. Kristeva emprunte le terme chora à Platon qui l'utilise dans le Timée pour désigner ce lieu ou réceptacle indéterminé à partir duquel l'ordre du Même et de l'Autre (de la matière soumise à la géométrie) sera ordonné par le dieu identifié à l'Agatbon, au Bien ${ }^{17}$.

Le rythme de ces premières scansions est la condition de possibilité de l'avènement du jeu des différences plus complexes qui s'élaborent par le langage et, via ce dernier, assurent l'entrée du sujet dans l'ordre de la culture. C'est sur ce premier fond toujours déjà différencié que vient en effet se greffer le jeu de la différence s'articulant selon une logique de la lettre ou du signifiant. Par signifiant, on entend depuis Lacan, relisant Freud, ce qui dans l'expérience première du langage advient comme matière sonore détachée du référent, mais lié cependant à l'appel et au manque déterminant la survie de l'infans ${ }^{18}$. Ce détachement fait en sorte que le sujet, avant de saisir la portée référentielle du langage, expérimente d'abord sa position dans le désir par le jeu babillant des sons qui vient rompre la surface et le silence du monde. Le babil, ou le jeu du signifiant, est en ce sens, pour le sujet, le jeu de cette différence par où le manque persiste à se dire depuis un désir qui n'est jamais absolument comblé. Entre le signifiant et le signifié, il y a une barre qui tient lieu de refoulement et de coupure, le procès de la signification étant aussi de l'ordre de l'inconscient ${ }^{19}$.

En cela, on peut dire que le langage et le processus de son acquisition ont valeur de paradigme dans cette ontogenèse de l'espèce humaine;

17. Platon, Timée 49 c, op. cit.

18. Voir Jacques Lacan, "L'instance de la lettre dans l'inconscient ou la raison depuis Freud", Écrits, Paris, Seuil, coll. "Le Champ freudien ", 1966, p. 493-527.

19. Comme le dit d'ailleurs Denis Vasse: "Ce qui est premier n'est pas un signifiant, c'est l'articulation signifiante, la coupure, le réfléchissement ou la réflexion où vient se prendre le désir du sujet. Ce qui est originaire n'est aucune trace, c'est l'activité de tracer. C'est pourquoi l'origine ne se signifie jamais que brisée et diffractée dans la césure originaire du réseau signifiant, là où se repèrent la parole et le désir du sujet. À cette césure, toutes les césures du langage renvoient "(Denis Vasse, op. cit., p. 92). 
le mot (et avec lui le sens) n'advenant que par le jeu des différences phonématiques, l'enfant expérimente en babillant toute la gamme des sons avant de pouvoir en fixer la signification au fur et à mesure qu'il trouve ses repères dans la position cedipienne. C'est donc par le jeu babillant de la voix que ce fond pulsionnel se donne à entendre non seulement dans sa jaculation première, mais aussi en ce qu'il est indiciel d'un appel ou d'une demande faisant du corps un corps déterminé par la loi du désir. Le babil est cette première phase exploratoire où le langage apparaît pour ainsi dire dans sa pure matérialité (signifiant); sa plasticité le désigne à cette place pour jouer, par la voix, le rôle d'un équivalent général pour la pulsion. Cette voix exploratrice est d'une certaine façon l'un des tout premiers corps du sujet qui fait du matériau phonique l'objet de tous les affects: de l'agressivité (cris, pleurs, vociférations) à la béatitude du gazouillement (un léger sifflement), toute la gamme des affects peut en effet prendre forme dans le babil. La voix a pour ainsi dire une fonction phatique en ce qu'elle fixe le corps dans l'horizon d'une demande qui le fera corps désirant. Comme a pu l'analyser Serge Leclaire, le corps est également travaillé par le jeu de cette différence d'où un sujet se saisit ${ }^{20}$. À l'opposé de ce corps érotisé par le jeu de la différence, nous retrouvons le corps-éponge ou le corps sans limites (lisse) du schizophrène qui ne parvient pas à situer son désir à l'égard de l'autre et du monde.

C'est par le stade du miroir, enfin, que ce corps trouve à s'anticiper sur le mode spéculaire d'un leurre inaugural par où il se saisit à la fois comme corps unifié et cependant séparé ${ }^{21}$. Cet autre effet de coupure sépare le sujet de la chora en même temps qu'il le voue au jeu de la différence ou à la mascarade de l'imaginaire. La seconde coupure, qui vient séparer le corps pulsionnel prenant plaisir à s'agglutiner à la mère archaïque - la chora -, est l'assomption de la castration de la mère et de la symbolisation de la différence des sexes comme accès au désir et à la jouissance. Le fort-da est le jeu de cette scansion entre maitrise et dessaisissement de l'objet, apparition et disparition, jeu du manque qui inscrit le sujet dans l'horizon ou l'appel de tout désir ${ }^{22}$. Sur ce point, le sujet anticipe désormais son rapport à la mère sur le mode du manque (et non du corps plein satisfaisant à toute demande), symbolisant du coup la place du père et son nom comme instance de la transmission d'un interdit et d'une loi. La reconnaissance du père n'advenant que par un effet de la parole, s'ouvre cette place qui, par l'interdit de l'inceste, est celle de la loi en tant que lieu de séparation ou de différenciation des sujets. C'est pourquoi, enfin, le nom propre fonctionne comme repère du sujet dans l'ordre

20. Serge Leclaire, Psychanalyser. Un essai sur l'ordre de l'inconscient et la pratique de la lettre, Paris, Seuil, coll. "Points", 1968, p. 55-96.

21. Jacques Lacan, "Le stade du miroir ", op. cit., p. 93-100.

22. Sigmund Freud, "Au-delà du principe de plaisir", traduction Jean Laplanche, J. B. Pontalis, Essais de psychanalyse, Paris, Petite bibliothèque Payot, 1981, p. 49-56. 
symbolique, repère qui donne cependant interminablement sur le défilé imaginaire de ses identifications selon la structure particulière du désir qui le détermine ${ }^{23}$.

Cette conception du langage comme jeu exploratoire des différences par où adviennent un sujet et une loi permet peut-être de penser ce qui se produit dans le travail de l'écriture pour Gauvreau. On sait que ce qu'il qualifie de "langue exploréenne" est précisément ce travail à même le corps du signifiant. Lorsqu'on lit cette lettre du 13 avril 1950 où Gauvreau expose son projet poétique, on remarque d'abord que c'est au nom de Freud, de l'inconscient et du désir que l'écriture peut et doit s'engager dans la voi(e)x de l'exploration ${ }^{24}$. En cela, bien sûr, cette nouvelle poétique qu'il nomme l'automatisme surrationnel s'inscrit dans la foulée du Manifeste du surréalisme de Breton, avec cette différence toutefois que la poétique de l'image gauvrienne veut aller plus loin en jouant sur la matérialité même de la langue. Cette poétique n'est pas une méthode, insiste$\mathrm{t}$-il, mais une certaine disposition du sujet à l'égard d'une autre écoute de l'inconscient. Influencée par l'automatisme pictural d'un Paul-Émile Borduas, la poésie de Gauvreau vise notamment à traiter le langage comme une matière plastique; en cela, la lettre, la syllabe, le son sont d'entrée de jeu des objets dont la matérialité est signifiante et ce, bien avant que tout cela ne s'articule et ne se décode dans l'ordre grammatical et référentiel d'une langue. Bref, la matérialité du signifiant, support où le sujet expérimente son désir en se faisant processus rythmique, précède la visée référentielle de l'ordre du signifié: "L'image rythmique, comme le clame le jeune Gauvreau, se déduit de cette constatation déjà ancienne que "tout son est une onomatopée". L'image rythmique est la charpente fondamentale, fatale, sans laquelle l'existence d'un objet est impensable. [...] Qui dit son dit rythme [...]. Qui dit lettre dit son. Il est impossible d'avoir une poésie sans rythme [...]"; et plus loin, dans cette même lettre, il ajoute: "Il n'y a pas de syllabe qui ne soit la partie intégrante d'avalanches de mots. Ce que cette syllabe représente, c'est précisément ce qu'il y a de commun chez chacun de ces mots. [...] Les syllabes, les mots, sont des valeurs, des teintes, ce ne sont pas les laquais de quelque monstre abstrait inexistant 25 !,

23. Voir René Major, Le Discernement, Paris, Aubier, coll. "La psychanalyse prise au mot", 1984.

24. Claude Gauvreau et Jean-Claude Dussault, Correspondance 1949-1950, Montréal, l'Hexagone, coll. "EEuvres de Claude Gauvreau ", 1993, p. 285-306.

25. Ibid., p. 293 et 299. On peut lire dans cette même lettre l'essentiel de son projet poétique; ainsi, ce passage où il est explicite que la matière verbale, le jeu du signifiant, est l'expression du désir jusque-là indicible du sujet : "La poésie joue donc constamment sur l'analogie. Un poète, par exemple, éprouvera un état psychique singulier, un état qui ne se répétera pas - cette ambiance psychique est pour lui la réalité poétique. Seulement, cette réalité, comme elle est sans précédent et sans identité, il ne pourra jamais la traduire par un signe conventionnel simple; cependant, cette réalité, comme 
S'affirme ici, donc, l'antériorité du rythme par rapport à l'élaboration du poème ou de la parole. Le rythme serait à comprendre comme ce qui, dans la constitution du sens, est non seulement avant lui mais le conditionne; le rythme serait ainsi ce fond toujours mouvant du langage en lien direct avec le corps du sujet ${ }^{26}$. Ce qui est visé par cette poétique, c'est la traduction des rythmes du corps enfouis dans la mémoire du sujet. Une autre logique que celle du discours analytique ordonne ce procès poétique puisqu'il ne s'agit pas en effet de discerner des éléments plus ou moins stables appartenant à la langue, mais de retrouver à même la matière verbale les différents rythmes, sinon les drames, les nœuds et les cris qui agitent le sujet. L'essentiel réside ici en ce que le mot cesse d'être le terme premier du dire poétique, pour laisser prédominer la lettre, le son, la syllabe, le phonème. On pourrait dire qu'il s'agit en somme d'une poétique du corps langagier, bien que toute poésie joue explicitement aussi avec de savants effets de signifiant. En fait, l'image rythmique, mémorante, transfigurante ou exploréenne joue au maximum de la plasticité dū langage; toute la gamme des dissolutions du mot ou du nom est possible de même que, simultanément, toutes les reconstructions ${ }^{27}$. Ce double processus de dissolution et d'agglutination fait en sorte que le langage devient un corps protéiforme susceptible d'être investi de toutes les significations et de tous les désirs du sujet. Il y a là sans doute une intuition juste quant à ce qui fait du langage et de la voix un corps, mais cet effacement du nom n'est évidemment pas sans créer une sorte de vertige. Il y a là le retour de cette très ancienne rêverie d'un langage parfaitement motivé effaçant l'arbitraire du signe. Il n'est donc pas étonnant de retrouver à la base de cette poétique élaborée par Gauvreau la formule (mise

elle est une réalité psychique humaine et qu'il n'y ait rien dans la mentalité humaine qui ne vienne directement du cosmos, le poète peut (spontanément), en prenant des réalités simples ou des fractions de réalités simples et répandues, constituer une nouvelle réalité dont la totalité sera une équivalence adéquate de l'ambiance psychique initiale. [...] Mille fractions d'analogies, juxtaposées ou fondues ensemble, figureront une réalité unique (et parfaitement connaissable par les sensibilités alertes chez lesquelles on suppose assimilées les mille analogies courantes). [...] Je dis donc que l'image rythmique n'est pas exclusivement musicale; elle se traduit par le son, mais non pas en tant qu'il frappe l'ouie, mais en tant qu'il est convoyeur d'analogie. Je conçois donc l'image rythmique en tant qu'essentiellement une onomatopée. Évidemment, il faut élargir le terme onomatopée au-delà de toutes les frontières admissibles jusqu'ici. Il n'y aura pas onomatopée uniquement lorsqu'un objet circonscrit et défini sera suggéré par une imitation simpliste - mais il y a aura onomatopée chaque fois que, par un rythme verbal, une bribe de réalité (nouménale ou phénoménale) ou un climat psychique pourra être conçu." (Ibid., p. 295-296)

26. Henri Meschonnic a également élaboré, dans son livre Critique du rytbme. Anthropologie bistorique du langage (Paris, Verdier, 1982), une théorie du rythme comme antérieure et condition de possibilité du signe, du langage, du poème.

27. Pierre Popovic a bien analysé la poétique gauvrienne dans son livre La Contradiction du poème. Poésie et discours social au Québec de 1948 à 1953, Montréal, Balzac, coll. "L'Univers des discours", 1992, p. 255-327. 
entre guillemets mais dont on ignore la référence), "tout son est une onomatopée ", laquelle nous ramène à la question de la motivation du signe. L'onomatopée serait ici le nom de cette langue mimant le corps et faisant fi de l'arbitraire par où, et au-delà de toute forme d'idiosyncrasie, la langue fonctionnerait comme un équivalent général pour l'échange et la rencontre de l'autre ${ }^{28}$.

Le langage exploréen de Gauvreau est certes une altération de l'ordre des unités linguistiques, lexicales et syntaxiques, mais il est aussi exploration jubilatoire des éléments différentiels prévalant à la formation du signe. En cela, on peut dire que le sujet jouit continuellement de ce jeu différentiel mais à la manière d'un fétichiste: déni de la castration qui est déni de la socialité arbitraire du code de la langue en même-temps qu'il ne cesse par ce jeu d'en mimer la forme et la venue. Paradoxe ou doublecontrainte? Il y a là à la fois maîtrise du jeu différentiel et dessaisissement de l'ordre de la langue. Le sujet est en quelque sorte capté ici dans son babil ou dans le chant solipsiste d'un plaisir qui tient à distance la jouissance, la rencontre de l'autre, bref, la castration. Jeu masturbatoire aussi, érotomaniaque, où le sujet cherche à se donner un corps de désir et jouit dans l'hallucination ${ }^{29}$. De ce point de vue, le babil exploréen serait le jeu d'une différence n'opérant pas, laissant le sujet à la rêverie d'un chant qui lui donne le simulacre d'une toute-puissance, mais qui ne peut manquer aussi de faire en sorte qu'il déchante, retombant lourdement dans la prostration, la pétrification, la catatonie, là où, enfin, la pulsion de mort le domine ${ }^{30}$. C'est pourquoi on peut dire que l'exploréen est le babil à la fois triomphant et véhément d'un appel à la différence; triomphant, en effet, dans la mesure où le sujet se donne d'abord tous les possibles,

28. Comme le note cependant Henri Morier dans son Dictionnaire de poétique et de rbétorique (Paris, Presses universitaires de France, 1982) sous le terme onomatopée: "Le canard français fait "coin! coin!", le canard allemand "was! was!". "C'est dire tout ce qui reste d'irréductiblement arbitraire dans l'usage de la langue.

29. Comme l'a déjà noté Pierre Nepveu: "Je ne suis pas loin de penser que l'onanisme est une des clés de cette œuvre " "Note provisoire sur les œuvres créatrices complètes", Lettres québécoises, $\mathrm{n}^{\circ} 7$, août-septembre 1977, p. 17). André Brochu retrouve cette intuition dans son analyse d'un poème des Boucliers mégalomanes, "Lettre sur la lettre en poésien, Revue d'esthétique (Collages), Paris, 10/18, 1978, p. 132-149.

30. C'est aussi ce babil mortifère que décrit Julia Kristeva: "Chez le sujet parlant, le fantasme articule cette irruption de la pulsion dans l'ordre du signifiant: il le bouleverse, et déplace la métonymie du désir qui agit le lieu de l'Autre en une jouissance désinvestissant l'objet et revenant vers le corps auto-érotique, tandis que la défense langagière livre ainsi son ambiguité : la pulsion de mort qui sous-tend. [...] C'est ainsi que nous pouvons penser d'ailleurs toutes les "déformations" poétiques de la chaîne signifiante et de la structure de la signification: elles cèdent sous l'assaut des "restes des premières symbolisations" (Lacan), c'est-à-dire des pulsions que la phase thétique n'a pas pu relever pour les enchaîner en signifiant/signifié. De sorte que tout dérèglement de la "censure d'ordre social" qu'est la censure signifiant/signifié témoigne entre autres, sinon avant tout, d'un afflux de la pulsion de mort qu'aucun signifiant, aucun Autre, aucun miroir et aucune mère n'ont pu satisfaire." (op. cit., p. 47) 
explorant sans limites le spectre sonore depuis la position de maitrise d'un poète onomaturge; véhément, cependant, puisque cette exploration est en même temps le malheur d'un sujet qui demande vainement à être reconnu comme le poète génial d'une nouvelle fondation ou instauration de la langue et du monde. C'est ce lien d'hainamoration qu'entretient Gauvreau avec la langue et qui le pousse à s'y livrer à un continuel jeu de destruction et de reconstruction.

Malgré cela, l'antériorité affirmée du rythme ou de l'image rythmique n'efface pas pour autant, on va le voir, le procès référentiel qui reste, à divers degrés, dans l'horizon de lecture de cette expérience d'écriture. Relisons, encore une fois, ce Jappement à la lune:

ghédérassann omniomnemm wâkulé orod écmon zdhal irchpt laugouzougldefterrpanuclémenpénucleptadussel ferf folfoufaulô farmurerr a clô dzorr (OC, p. 1498).

Cela tient-il du cri ou du hurlement irréductible à l'ordre d'une grammaire et d'une poétique? Ou bien ce magma de phonèmes est-il écrit, et lisible malgré tout dans l'horizon d'une langue? Bref, malgré l'opacité première et apparente de ce magma de consonnes et de voyelles, le lecteur ne continue-t-il pas de lire, de traduire, ce qui se présente à lui comme du texte depuis l'horizon structurant de sa langue; à savoir, d'abord le jeu des différences phoniques comme première articulation du mot et du nom, puis de la phrase avec son ordre syntaxique? N'y a-t-il pas là un ordre aussi minimal soit-il qui puisse être assimilé à une prosodie?

Comme on peut le lire dans des études sur le phénomène de la glossolalie, ces langues exploratoires qui, à première vue, semblent totalement arbitraires ou inconnues, sont, malgré tout, “inventées" à partir de la langue du sujet; de plus, ce jeu exploratoire tend à l'homophonie, c'està-dire qu'il cherche à se stabiliser autour d'un certain nombre de sons, sinon d'un unique son ${ }^{31}$. Il y à donc là, par-delà le déchaînement du jeu mortifère de la différence, la mise en scène d'une stabilisation imaginaire des amalgames phonématiques telle qu'on la retrouve dans la langue où les mots et les noms incarnent des points d'inscription stables. On pourrait dire ainsi qu'il y a là une manière de mimer, par-delà le vertige de l'exploration, l'inscription d'un nom qui fixe le sujet à l'ordre du langage. Bref, le jeu de la différence parvient à s'arrêter, d'une certaine manière, au fur et à mesure que le sujet mime l'effet de discernement du nom ${ }^{32}$.

Du point de vue phonématique, on remarque ici que le continuum tend, sinon à l'homophonie, du moins à une forte redondance; ainsi, la

31. "La forte redondance des sons et leur réitération en chaîne est donc l'une des caractéristiques phoniques les plus apparentes de l'énoncé glossolalique, qui tend ainsi vers l'homophonie ", Jean-Jacques Courtine, "Les silences de la voix. Histoire et structure des glossolalies ", Langages, n ${ }^{\circ}$ 91, septembre 1988, p. 20.

32. Voir René Major, op. cit., p. 43-81. 
récurrence des phonèmes suivants : é assa nn omom ni nem mm o m mon o au ou ou pan pén ou au o rr. Le jeu d'opposition entre la labiale consonantique $m$ et la vocalique o produit, malgré l'apparent désordre, une fréquence qui se structure autour de ces effets de répétition. Cette récurrence mime en fait, par-delà tous les possibles de ce jeu différentiel, les effets de stabilisation phonématique inhérents à la constitution d'une langue; en cela, l'écho d'un même phonème au fil d'une exploration phonématique est comme le désir de former un nom contre le vertige mortifère de l'indifférenciation. Le phonème en écho est la trace de ce désir de nom par où le jeu de la différence peut prendre sens sans que le sujet ne s'y perde sur le mode d'une psychose où tous les noms se valent et ne valent rien quant à une possible rencontre avec l'autre ${ }^{33}$. L'exploration de la différence en vient ainsi à se stabiliser autour de certains phonèmes laissant entendre par là que le langage est aussi déterminé, à ce niveau, par ce procès de stabilisation imaginaire autour d'un et de plusieurs noms, véritables stases d'où rejaillit le jeu de l'identité et de la différence. En se faisant écholalie, le continuum phonématique montre que le poète exploréen reste ici assujetti à l'ordre oppositionnel du nom et du sémiotique. L'écholalie est la trace de cette fixation au nom qui advient dans le jeu du même et de l'autre.

D'un point de vue formel, dans la mesure où l'on cherche à reconnaître des niveaux plus élaborés du discours - syntaxe, rhétorique et ordre du récit -, on peut dire d'abord que ces amalgames miment, par le jeu de scansion des espaces blancs, le rythme sur le mode d'un phrasé. La scansion des espaces mais aussi le jeu alternatif de ces amalgames entre des temps longs et des temps courts rappellent les différents éléments constitutifs de la phrase et de sa syntaxe (le groupe sujet et le groupe complément s'avérant plus longs que le groupe verbal). Ainsi, cette glossolalie peut-elle être classée selon sa densité temporelle. Mais la succession de ces temps constitue-t-elle un ordre analogue au déroulement d'un drame ou d'un récit? Sans lexicalisation, il est certes impossible d'attribuer à la succession de ces amalgames un sens déterminé. Malgré tout, le rythme est déjà entendu par le sujet dans l'horizon d'une sémantisation. L'amalgame final, par exemple, est bref, aporétique ou sentencieux: sa brièveté peut s'entendre comme le dernier mot qui tranche dans le défilé exploratoire des noms, bien que le $r$ soit par excellence le phonème de la réverbération et de l'écholalie: en cela, cette vocalisation raconterait encore le jeu dramatique de cette tension entre l'appel d'un nom et sa fuite continuelle dans le jeu des possibles phonématiques.

Il est sans doute difficile d'attribuer un discours de motivation entre le phonème et le corps pulsionnel, bien que la fine oreille d'un psychanalyste

33. Voir Serge Leclaire, "Les mots du psychotique", Rompre les charmes. Recueil pour des enchantés de la psychanalyse, Paris, InterÉditions, 1981, p. 29-45. 
puisse en repérer l'incidence ${ }^{34}$. Cela relève moins d'une science (d'une grammaire) que d'une intuition de la langue qui pourrait, en tant que système de différences, servir de relais à un autre système de différences; ainsi le discours sur le son est-il toujours déjà métaphorique, comme l'attestent les définitions de la grammaire; entre le dur et le liquide s'ordonne une opposition culturelle où l'imaginaire de la dureté s'oppose à celui d'une matière moins opaque et plus transparente. C'est dire que la description de la langue est ici non seulement une description corporelle, mais une description d'un système d'oppositions qui se métaphorise dans le champ de la culture. Dans le cas qui nous occupe ici, on remarque qu'il s'ouvre avec un $g$ ("ghé "), soit un phonème ou une consonne occlusive palato-vélaire, lequel son est l'un des plus gutturaux. De là, cette "voix de gorge" suggère l'archaïque de la langue, sa profération originaire. Ce continuum exploréen s'arrête sur le $r$ ("dzorr"), une consonne continue liquide (vibrantique) dorso-vélaire qui se situe à l'extrémité du spectre des sons consonantiques. Entre $g$ et $r$, il y a donc comme le parcours hyperbolique du spectre sonore par où le sujet mime le drame du fermé (occlusif) et de l'ouvert (continu). L'opposition aussi, dans la suite de ces amalgames phoniques, du $g$ et du $f$ s'articule également sur celle qui existe entre l'occlusive et la continue fricative labio-dentale.

C'est ainsi que, à même le corps de la langue, le sujet résiste pour ainsi dire à l'étouffement, à l'avalement, au mutisme virtuel d'une occlusive qui se referme sur elle-même. On reconnaît, vers la fin et comme encryptés dans cette vocalise, quelques mots qui sont là telles les traces de ce drame de l'enfermement: fou, mur et clos (clô) redisent en effet l'occlusive qui cherche à se frayer un chemin. En cela, ce sont des traces à l'intérieur d'amalgames résultant, comme dans le rêve, d'un travail de déplacement et de condensation. Enfin, omniomnemm peut être traduit par "tout-homme" ou "homme-négation", ce qui implique justement une opposition ou un conflit quant à la capacité qu'a le sujet de se repérer dans l'ordre d'un nom. C'est par là également le nom d'un certain seuil de l'humanité qui apparaît pour ainsi dire avec véhémence dans ce qui est titré comme étant un jappement, un aboiement de chien, du corps langagier animalisé. La trace de ce signifiant, om, insiste donc le long du spectre sonore pour former un nœud et mimer les effets stabilisants de la nomination. Ce signifiant om est d'ailleurs présent et de manière très significative, on le verra, dans la tragédie intitulé L'Asile de la pureté.

L'appel de la différence est finalement l'appel d'un nom qui tranche dans le défilé exploratoire des phonèmes. La langue est ainsi simultanément en état d'éclatement et de recomposition, sinon de fondation. Et

34. Voir François Peraldi, “Corps du texte et corps érotique ", Texte, n 4, 1985, p. 177-189. 
c'est bien là le paradoxe de ce discours: le sujet rêve à la pure profération et reconnaissance d'un nom au fur et à mesure qu'il expérimente la déliaison du code de sa langue. Il se déchaîne dans l'appel des signifiants en même temps qu'il appelle à la pureté d'une langue garante de son inscription dans la loi (du nom). Le nom est pour ainsi dire anagrammatisé (crypté) dans le flux de cette vocalisation qui l'enferme au moment même où il en appelle à la pureté. L'écholalie est cet effet de réverbération par où s'entend de loin en loin l'appel à la stabilisation imaginaire.

Cette analyse se fonde sur le fait que, étant assujetti à l'ordre du langage, le sujet ne s'anticipe que dans l'horizon de la phrase et de son inscription dans la chaîne signifiante. En cela, la glossolalie est encore la trace de l'inscription du sujet dans une chaîne, bien qu'elle soit la manifestation d'une difficulté pour le glossolale à s'y inscrire. L'écoute, l'écriture tout autant que la lecture, ne peut échapper à un certain procès de sémantisation inhérent à la condition même du sujet dans la langue, qu'il soit glossolal ou non. L'ordre de la lettre le lie au nom, au thétique, ne serait-ce encore que dans le désordre exploratoire de ces vocalisations; chapelets, grappes, sporades, archipels, îlots, les noms se répartissent selon des enchaînements plus ou moins déterminés. Le texte gauvrien met en scène le jeu d'un contrepoint entre la glossolalie et le phrasé à la fois néologiste et syntaxique. Le second moment mime l'intelligibilité de l'énoncé et vient soutenir la pertinence linguistique de la trame glossolalique pour donner l'illusion que la lecture peut être performée selon le protocole habituel. Ce cadre ou ce protocole de lecture permet de fixer le sujet à l'ordre du langage malgré le dérapage exploratoire de la voix à la recherche de ses lettres et de son nom. Peut-être l'exploréen n'est-il aussi en cela que l'ultime point d'ancrage d'un corps dans l'ordre du langage, aussi véhément soit-il, contre la menace d'un silence absolu mimant la folie et la mort, comme on peut d'ailleurs le lire très souvent dans le théâtre, ne serait-ce que dans L'Asile de la pureté.

\section{Le chant et le chantage du poète maudit ou L'Asile de la pureté}

L'Asile de la pureté se présente comme une tragédie. L'intrigue en est désespérément simple: un poète, Donatien Marcassilar, décide de jeûner suite à la mort de sa bien-aimée actrice, Edith Luel; il ne renoncera à ce jeûne que lorsque toute la communauté artistique l'en aura supplié. Il n'y a pas là d'intrigue, de péripétie ou d'énigme à déchiffer tant sur le plan de la forme que sur celui du thème.

De l'exploréen comme babil exploratoire du corps qui n'est pas encore séparé de la mère, à la mise en scène du drame du poète maudit drapé dans la toute-puissance de son verbe, il n'y a en fait qu'un seul et même procès qui concerne en définitive un certain ratage pour ce sujet quant à la symbolisation de la loi. Lisons le poème que récite Donatien 
Marcassilar, poète-héros qui se laisse mourir de faim pour se venger diune société qui ne le reconnaît pas:

D. Marcassilar. — "Les trois suicides d'Ocgdavor Pithuliaz", dernier effort poétique de Donatién Marcassilar. [...]

(récitant) - «Rouge. Rouge. Rouge. Rouge brique.

Bzzddd - Bzzddd - Allhiou-cou-coumh!

Les fenêtres, alahi-dizi-dagalaghli - s'ouvrent sur un meurtre semi-vertical.

La poisse grogne.

Dans l'ultime agronomotte des soupirs d'agonie - alass - splott -

ippo-it-appott-plott-klott!

Un homme a dit adieu.

Grizz-Glouz-out.

Plout-voutt-deproutt-kizzegglakk-akklott-pott-môott!

Un homme a dit a dieu à la vie.

Vie de chat. Klyst-kyrryst-ghiss-mirryst.

Bézzzéroum. Tourrédoum. Apppitoum. Gléazzazza.

Rrréh - ohhhé - yyyoumm. Tattt.

Un homme - mort. Il est.

Deux fois. Tattazzic-hic-zic. Grrriimm.

Un homme - louc.

Vertèbre - tibaza-baza-za.

Un soupir........ de Mort.

Il y a - hic-aboudzou. Un homme-tarabom — patablom — un homme bomm.

Trois.

X. C'est final.

Un homme. Plom.

Mort. Il

Applomm. Un homme.

Il est.

Applomm. Pomm.

Il est.

Un honme.

Zic.

Ic.

Bic.

Un Homme.

Il est. Arrazzoum.

Un homme. Zoum-arra. Tippata.

Il est. Un homme. Il est. Lougk.

Un homme. Il est.

Mort.

Il est.

Zoum.

Trabbazza.

Ikkakkla. Vic. Trouppouc. Homme. Lik. Il est. Zoumpi. Vic.

Zic. Broutttt - thah!

Il est... Trois.

Il... Bic. Vlic.

Il est... Oupslâh!

Il est... Oungh. 


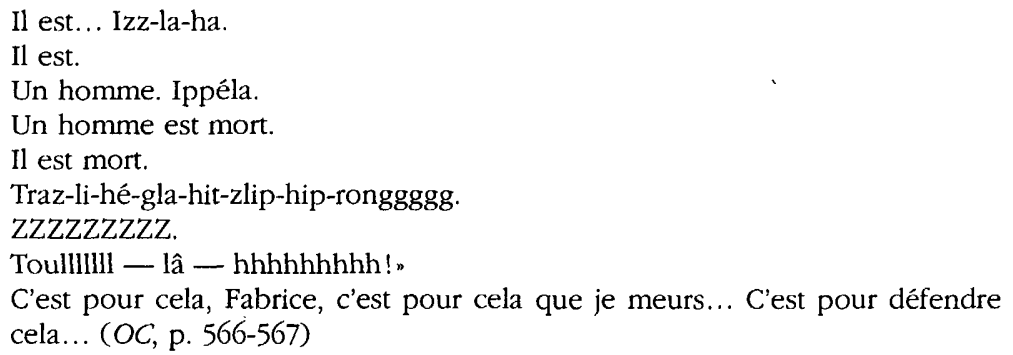

L'accent sur les lettres et les phonèmes plutôt rares en français $(k, z$, $y, g, b)$ a pour effet de souligner l'étrangeté de cette langue inventée. L'étrangeté de la lexicalisation laisse malgré tout passablement intact l'ordre du discours poétique comme jeu de règles prosodiques; assonances, allitérations, anaphores. En fait, nous lisons là une litanie par le jeu de répétition des mêmes propositions ${ }^{35}$. Le lexique étant parfois étranger à l'ordre courant de la langue, le jeu de la métaphore comme usage particulier de la prédication à l'intérieur d'un monde préalablement classifié, n'opère pas; c'est plutôt par métonymie et par paronomase que le texte s'engendre et se donne comme sa loi de composition. Cet entrelacement de babil et de francais a plusieurs effets: d'abord, la norme de l'un renforce l'étrangeté de l'autre; ensuite, il arrime le pulsionnel à un récit, aussi minimal soit-il; enfin, le babil pulsionnel a un effet de trouée sur le langage conventionnel.

Or, mis à part les quelques syntagmes du début, c'est le signifiant "homme" qui se donne pour point de référence, de butée, de toutes ces explorations verbales. Ce signifiant forme un nœud sur lequel vient s'agglutiner un ensemble de déclinaisons qui en portent l'écho. Comme si le jeu du dérapage écholalique retombait sur ses pieds pour mieux se faire l'appel de ce que le nom recouvre de sens. C'est dire, par-delà son aspect transgressif, que ce discours reste arraisonné à un signifiant, lourd de signification. "Un homme" est le générique pour nommer l'espèce humaine et l'identification sexuelle. Tout se passe donc comme si l'étrangeté de cette langue revenait s'ancrer dans une classe d'objets surdéterminée sur le plan de l'histoire. Cette profération exploréenne du nom de l'homme ne retrouve ici qu'une règle de rhétorique déjà connue, l'assonance, et le nom qui subsume tous les noms dérivant au fil de cette exploration. De même, la scansion des "il est" souligne cet appel identitaire du sujet, le verbe "être" étant là encore comme au fondement du geste prédicatif par lequel un sujet se désigne parmi les multiples noms du monde. Malgré toutes ces déclinaisons exploréennes, le nom peut se

35. Comme le remarque Michel van Schendel dans son article "Eulalie ou la malédiction du tant-à-dire. À propos de Claude Gauvreau ", Rebonds critiques I. Questions de littérature, Montréal, l'Hexagone, coll. "Essais littéraires", 1992, p. 208-218. 
défaire puisque le nom "homme" reste discernable. La scansion des "il est" et des "un homme " forme ainsi un nœud stabilisant le mouvement de dissémination de la vocalisation exploréenne. C'est en cela aussi que le sujet, malgré la force délirante de son verbe, reste attaché au procès nominal inhérent à la langue. Comme si le nom, à l'instant pathétique de cette mort, mettait un terme au déchaînement imaginaire de cette parole.

Mais L'Asile de la pureté s'achève lorsque le personnage de Claude Gauvreau apparaît sur la scène, interrompt le drame pour convaincre Donatien Marcassilar d'abandonner son jeûne. L'auteur apparaît donc sur scène pour arrêter une tyrannie, un chantage, et convaincre son propre personnage, son double, d'opter pour la vie. Tout se passe comme si le sujet se rappelait ici à l'ordre de son inscription symbolique, c'est-à-dire à son nom propre, pour s'arracher à la toute-puissance de son nom mythique qui le sacre poète maudit et incompris. Le nom de Claude Gauvreau vient donc trouer la trame du texte pour l'arraisonner à l'ordre qui le fait sujet de la filiation et du désir, tout comme le nom "homme" est le nom qui vient stabiliser le défilé imaginaire d'un sujet capté par le désir de sa mort. Entre la fiction et le réel de l'inscription symbolique, le nom de Claude Gauvreau fait ainsi étrangement retour pour arrêter le jeu de la mort de celui qui s'enferme dans la rêverie de son babil mortifère et mégalomaniaque ${ }^{36}$. Comme s'il n'y avait ici que le réel du nom propre pour arrêter la tyrannie d'un imaginaire qui a perdu ses repères symboliques $^{37}$.

Quand on relit le projet poétique de Gauvreau, de même que l'imaginaire de ces mises en scène répétitives où se joue le drame du poète incompris, maudit et agonisant, on comprend mieux cette tension paradoxale entre l'exploréen et la pose hiératique, solennelle et sentencieuse du poète onomaturge qui doit régner sur le monde. Entre la parfaite maîtrise de l'un et le babil solipsiste de l'autre, il n'y a qu'un seul et même sujet divisé entre le plaisir d'une langue idiomatique qui serait fusion avec la mère et le désir d'appartenir corps et âme à la loi comme lieu d'assujettissement du sujet à l'arbitraire de la langue. C'est, semble-t-il, cette double voix contradictoire qui se donne continuellement à entendre chez Gauvreau: langue à soi mais qui porte l'appel véhément d'une loi. D'où

36. Voir Claude Gauvreau, L'Asile de la pureté, OC, p. 601-604.

37. On remarque ce vertige du sans-nom dans Beauté baroque (OC, p. 379). L'hérö̈ne est sans nom propre, mais elle est pour ainsi dire comblée de surnoms; son nom est ainsi comme une perpétuelle périphrase. Roman sans intrigue ou action véritable, il s'agit plutôt d'un texte qui est une sorte de blason du corps féminin. L'objet du discours amoureux possède ici virtuellement tous les noms de la séduction; elle est l'Unique au nom infini. Le sujet n'apparaît donc pas ici, par son nom propre, comme sujet de la finitude, mais comme le nom infini qui d'emblée possède tous les noms. Le narrateur est ainsi capté, maîtrisé par le nom infini du désir et ne peut rencontrer l'autre comme ce sujet de la finitude dont le nom sert de point d'ancrage quant au désir. 
ce ton sentencieux et proverbial à même les formules exploréennes pour trancher dans le monologue et faire triompher enfin une loi, un dernier mot qui arrête le déchaînement de l'imaginaire. En cela, on peut dire que les déclamations exploréennes sont aussi, par la difficulté que soulève leur prononciation, des sortes de schibboleths ou de mots de passe par où le sens du-monde se révélerait enfin au sujet; elles relèvent aussi de l'holophrase dans la mesure où elles cherchent à se faire totalisantes du sens et, n'y parvenant pas, se relancent continuellement dans une autre phrase-exploration tout aussi hautaine et véhémente. Comme si le sujet ne cherchait jamais sa phrase (bien qu'il ne fasse que cela), mais nommait le monde une fois pour toutes, le maîtrisant, le remettant à sa place. Le sujet parle du haut de cette phrase solennelle pour trancher dans le verbiage, le charabia, le bavardage infini du monde et imposer un sens achevé. La phrase est le jeu de cette relance et de cette surenchère vers l'absolu d'un sens appelé enfin à trancher dans le magma de la parole inauthentique, le dernier mot qui est tout autant le mot de la fin que le mot d'ordre s'imposant à tous dans le monde. Mais ce jeu est sans fin comme ce désir est inassouvissable, le signe étant, d'entrée de jeu, le leurre d'une marque qui ne peut combler absolument le manque à être. L'illusion de cette demande de pureté tient à ce que le sujet anticipe son rapport au manque et à' l'ordre de l'originaire sur le mode d'un nom susceptible de le combler alors que ce nom ne peut que le situer dans l'ordre symbolique du désir comme manque et appel à la capacité de discernement de son nom pour un autre sujet. C'est pourquoi une phrase ne cesse d'en relancer une autre pour se faire successivement triomphe et véhémence du "sujet-de-la-langue". Il y a là un désir de fondation, d'instauration à partir de l'unique phrase qui ne saurait être une autre. D'où une langue de plus en plus totalisante (totalitaire), archaïque, mimant la profération originaire. Voix tutélaire s'imposant sur le monde pour vaincre la folie et l'inauthenticité de l'existence. Jeu tyrannique à travers lequel cependant le sujet se retient de tomber dans la psychose. Il éprouve le langage comme loi mais ne peut complètement l'assigner au lieu de l'Autre.

L'opposition pureté/corruption s'inscrit dans la langue et dans l'imaginaire mégalo-onomaturgique du poète maudit, incorruptible et rédempteur du monde. Plus le sujet explore la langue (dans ses effets de différenciation) sur le plan du signifiant phonique, plus il désarticule le code lexical pour privilégier d'autres amalgames et des métastases de diverses sortes qui prolifèrent dans tous les sens (sauf vers un langage déjà codé); le signifiant devient alors ce corps qui ne cesse de se corrompre à même le déchaînement phonématique, cela au fur et à mesure que le sujet s'expérimente et s'anticipe ouvert à (l'étrangeté de) tous les amalgames possibles; d'où la part du monstrueux chimérique qui revient hanter l'imaginaire. Sur le plan de l'exploration phonématique, le corps de la 
langue est donc celui d'une corruption dans la mesure où les éléments vocaliques et consonantiques s'altèrent ou se corrompent pour former d'inouïs phonèmes.

On pourrait interpréter l'exploréen comme étant cette corruption (violence à l'ordre du langage) par quoi le sujet laisse entendre autant l'incertitude de son ancrage symbolique que la violence de sa place, puisque ce jeu de corruption se fait jeu de massacre. Les effets de sublimation pour refouler la haine se donnent à lire dans ces appels tout aussi forcenés à la pureté de la mission poétique, la pureté de l'exploréen s'avérant celle d'une langue des fondations, de l'originaire et de la vérité. La pureté est ainsi le nom qui jette un voile sur le jeu de massacre exploréen de la langue. Rêverie compensatoire par où un sujet cherche à freiner le déchaînement exploratoire pour se refaire un corps inaltérable, incorruptible, pur et tel qu'en sa voix, il proclame sa maitrise du monde. Entre le babil mortifère et la pose hiératique du poète pur de tout trucage, il n'y a ainsi qu'un seul et même sujet qui cherche à se nommer dans sa langue.

Il n'est donc pas étonnant de lire chez Gauvreau tout un imaginaire du corps chimérique par où se remarque le ratage à symboliser la langue comme lieu de transmission du désir qui porte et fixe le sujet dans la loi. La chimère, et tout l'imaginaire de l'incorporation et du monstrueux, sont là au fil des pages pour dire les effets néfastes de l'indifférenciation du sujet capté par le babil et le désir de se fondre dans le corps archaïque de la mère ${ }^{38}$.

\section{La Ventouse anonyme ou le corps parasite/parasité}

D'emblée, nous imaginons un animal dont le corps tout entier se confond avec sa bouche ${ }^{39}$. Cette bouche est ici non seulement succion, aspiration, avalement, mais aussi corps qui vient parasiter un autre corps, l'altérer, l'absorber. Suivant l'échelle biologique de l'évolution et de la complexité du vivant, on peut dire qu'il s'agit d'une forme de vie animale plutôt "archaïque"; d'où son étrangeté, et le malaise que ce corps peut provoquer. Or, ce corps-bouche, nous n'avons cessé de le reconnaitre comme celui d'un sujet capté par le plaisir despotique de l'oralité première du babil. Cette ventouse en serait ainsi l'incarnation imaginaire; mais elle ne symbolise pas seulement le plaisir que prend le sujet à babiller, elle incarne également le sadisme dévorant d'un corps-bouche absorbant tous les phonèmes de la langue jusqu'à détruire les noms. C'est pourquoi sans doute cette ventouse est dite "anonyme"; elle est cette

38. "Le langage exploréen est ce qui se dresse sur la tombe silencieuse du père pour faire jouir la mère, jusque dans la mort..." C'est cette structure qu'analyse avec finesse JeanPierre Denis dans son article, "Claude Gauvreau: du tombeau du père au langage exploréen ". Voix et Images, $\mathrm{n}^{\circ}$ 54, printemps 1993, p. 483-494.

39. Voir OC, p. 1121. 
bouche aveugle engloutissant tous les noms du monde à sa suite. Elle est en quelque sorte le monde d'avant le nom, c'est-à-dire le monde tel qu'il ne s'accomplit pas depuis un procès généalogique où la reconnaissance de la différence sexuelle détermine le sujet dans le désir. Et ce monde d'avant le nom, appelé "ventouse anonyme", dit le nom symbolisant cet état limite qui capte le sujet. Enfin, ce corps-bouche est aveugle, tout comme celui qui possède cette ventouse.

Il est significatif que ce récit se déroule en même temps sous l'œil d'un dieu omniscient et tyrannique, Zeus Robinacle, qui se gausse du désastre du monde et des prétentions esthétiques de l'humanité ${ }^{40}$. L'œil du dieu domine ainsi le récit d'un martyr aveugle; en cela, Zeus Robinacle n'incarne pas ici le dieu de la transmission, du legs généalogique, de la filiation et du désir, comme le récit biblique du sacrifice d'Isaac par Abraham nous le donne à symboliser, mais celui d'une violence originaire qui serait là comme le contraire de l'avènement ou de l'instauration de la culture. On s'aperçoit ainsi que le poète exploréen, malgré la toutepuissance de sa place d'onomaturge, retrouve par les détours de l'écriture le désir de la loi telle qu'elle peut s'incarner dans le corps glorieux d'un dieu. Il ne retrouve pas cependant la figure d'un père séparant le sujet de la mère, mais celle d'un père tyrannique qui ne lui permet pas de symboliser sa place dans le désir. C'est en cela que le sujet est pris dans le vertige imaginaire d'un manque à symboliser son rapport à l'autre, mais aussi à symboliser son corps, et même à symboliser sa place dans l'humanité. Car ce qui revient là comme imaginaire d'un corps chimérique, c'est la question angoissante d'un seuil de l'humanité à établir pour le sujet, en l'occurrence d'un seuil qui n'advient que par la reconnaissance d'un sujet assujetti à la loi. Or, le corps chimérique serait comme l'effacement de ce seuil entre l'homme et l'animal, de la loi qui tranche et sépare ${ }^{41}$. Le tranchant de la loi n'opérant que difficilement, le sujet se cherche à travers toutes ses identifications imaginaires, se métamorphosant dans tous les corps. Ce vertige de l'imaginaire et du semblant apparaît d'ailleurs dans le texte à travers la figure du sosie qui, par effet de réverbération ou d'écholalie, est poussé jusqu'à sa limite dans l'expression "le sosie du sosie" consacrant encore le règne de l'indifférenciation. Ce monde dominé par le même ou le ressemblant est justement le monde d'une différence ratée, là où la loi et la loi du nom ne discernent pas le sujet dans son rapport à l'autre.

En ce sens, il n'est pas étonnant que ce récit soit aussi celui d'un "couple " qui, littéralement, ne se voit pas et ne s'entend pas. L'un soutient

40. "L'esthétique est-elle donc le seul remède aux maux? Qui donc l'inventa? Je ne me souviens pas d'avoir pensé à l'esthétique. Hein? Qui suis-je? Je suis Zeus Robinacle qui se pourlèche les babines au spectacle d'un univers déséquilibré à souhait. La claudication est le caramel de mon dessert " (OC, p. 1126).

41. On peut lire dans ce récit cette zoomorphie chimérique du corps de l'aveugle: ainsi, son " nez-perche", ses "bras nageoires", ses «moignons de primates", etc. (OC, p. 1124). 
l'autre dans son manque, mais ils ne se rencontrent pas véritablement. Et si la ventouse a pour fonction de servir de point de repère pour l'aveugle, force est de constater que ce qui s'érigera dans le dos de la sourde, c'est un temple de la virginité ${ }^{42}$. Le temple d'Éphèse, célèbre dans toute l'Antiquité, était dédié en effet à la déesse Artémis. Artémis y était représentée avec de multiples mamelles, incarnant la fécondité. Comme la Diane des Romains, elle est chasseresse, mais elle incarne aussi la virginité. Ce corps qui parasite un autre corps pour former une chimère est donc en définitive le montage imaginaire du corps virginal, intact quant à la jouissance qui ne peut s'avérer que par la différence. Cet "Imbécile de naissance", qui se fera poser la ventouse dans le front par le rationaliste d'où jaillira le flux exploréen, est d'ailleurs, littéralement, le surnom pour dire l'impasse d'un sujet quant à son désir. Le temple de la Reine-Abeille enkysté dans le dos de la sourde, c'est donc le corps chimérique de la toute-puissance féminine, intouchable et virginal, qui règne sans partage sur le monde. Corpstemple-bouche qui est, pour le sujet, le corps imaginaire d'une autre qu'il ne parvient à nommer que par ce qui la désigne comme inapte, justement, à l'écoute: sourde. Or, ce temple aussi s'effondre, là où toute inscription institutionnelle échoue à fixer le sujet dans le monde. Si la loi ou un quelconque mot d'ordre se laisse entendre dans le récit, ce n'est plus que par dérision ou peut-être comme un appel désespéré à joindre le lieu de l'autre: "il est impoli de montrer du doigt" (OC, p. 1129) est cette injonction qui vient ultimement ponctuer ce récit et dire, comme on peut le dire aux enfants, que l'autre existe et qu'il mérite considération. La politesse devient ici comme un autre seuil de l'humanité érigé contre le jeu de massacre ou la violence originaire d'un monde où règne l'indifférencié.

Ce. récit met donc en scène ce que l'on pourrait appeler l'intransitivité d'un langage qui cherche sa loi. Entre les bégaiements mécaniques de

42. Comme le note Jacques Desautels : "Artémis est une vierge farouche, dont le domaine est celui de l'espace sauvage, autant l'espace physique des territoires non ordonnés par les lois de la cité, non civiques donc, que l'espace temporel que vit chaque enfant jusqu'à son entrée dans le monde adulte, notamment par le mariage" (Dieux et Mythes de la Grèce ancienne, Québec, Les Presses de l'Université Laval, 1988, p. 242). Robert Graves souligne son lien avec Aphrodite: "La jeune fille à l'Arc d'Argent que les Grecs enrôlèrent dans la famille olympienne était le personnage le plus jeune de la triple Artémis, "Artémis" étant éncore un nom de la Triple-déesse-Lune; elle avait donc le droit de nourrir ses biches de trèfle, symbole de la trinité. Mais l'Artémis olympienne n'était pas uniquement une jeune vierge; ailleurs, à Éphèse par exemple, on adorait sa seconde personne, la nymphe orgiaque aphrodite, accompagnée d'un consort mâle. Ses principaux symboles étaient le palmier, le cerf et l'abeillen. Graves explicite le sens de ce dernier symbole ainsi : "Elle [Aphrodite Urania] tuait le roi sacré qui s'était uni à elle au sommet d'une montagne, à l'instar de la Reine-Abeille qui tue le mâle: en lui arrachant ses organes sexuels" (Les Mythes grecs, traduction de Mounir Hafez, Paris, Fayard, coll. "Pluriel", tome 1, 1967, p. 96 et 81). Il est donc tout a fait explicite que le travail de l'écriture retrouve ici non seulement le corps chimérique, mais le fantasme d'une émasculation comme rituel de fondation d'un socius dominé par la mère. 
Zeus Robinacle et le bourdonnement des abeilles, le sujet entend ici dans la cacophonie le ratage de son inscription symbolique. Ce bourdonnement serait d'abord comme la trace du désir d'un nom en tant qu'il en mime la stabilisation imaginaire; mais, en même temps, ce bourdonnement, qui est le bruit de fond ou le fondement du récit, n'est que la lettre assourdissante de ce qui n'est pas un nom et n'a pas franchi le seuil de l'humanité. Ce bourdonnement des abeilles (Bzzz) est donc là comme la "lettre assourdissante", le chant du même où ne prédomine plus que la lettre-son $\mathrm{zzz}^{43}$.

Et nous voilà ainsi revenus, d'une certaine façon, à notre point de départ. D'un sifflement, d'un bourdonnement à l'autre, de ces hommescigales à ces abeilles, ouvrières d'un temple aussitôt détruit, c'est encore, mais autrement, le récit d'une captation mortifère du sujet par le chant. Comme si, par un curieux renversement, le chant assourdissait l'oreille, non pas à la manière d'une dissonance, mais tel le sifflement infini d'une lettre à la surface des choses vides. L'étrange beauté du texte de Gauvreau, c'est de nous donner à rêver le langage comme un corps, comme un cri. Mais, c'est là, aussi, son drame et sa souffrance. Car s'il est indéniable qu'à lire ce plasticien de la langue nous sommes d'abord éblouis et emportés par son chant, n'est-il pas vrai, cependant, que ce chant a son envers là où, soudain, on peut entendre la voix discordante d'un poète qui cherche avec véhémence à faire entendre son nom?

43. Selon l'expression d'Éliane Formentelli dans son article "La lettre assourdissante: pour un "verbier" de Claude Gauvreau", Lectures européennes de la littérature québécoise, Montréal, Leméac, 1982, p. 327-343. 